\title{
Ideals of Largest Weight in Constructions Based on Directed Graphs
}

\author{
A.V. Kelarev ${ }^{1, a *}$, W. Susilo ${ }^{2, b}$, M. Miller ${ }^{1, c}$ and J. Ryan ${ }^{3, d}$ \\ ${ }^{1}$ CARMA Priority Research Centre, School of Mathematical and Physical Sciences, University of \\ Newcastle, University Drive, Callaghan, NSW 2308 \\ ${ }^{2}$ School of Computer Science and Software Engineering University of Wollongong, Northfields \\ Avenue, NSW 2522, Australia \\ ${ }^{3}$ School of Electrical Engineering and Computer Science, University of Newcastle, University Drive, \\ Callaghan, NSW 2308 \\ aandreikelarev-universityofnewcastle@yahoo.com, bwsusilo@uow.edu.au, \\ cMirka.Miller@newcastle.edu.au, dJoe.Ryan@newcastle.edu.au \\ * corresponding author
}

Keywords: balanced graphs, incidence semirings, semiring constructions, ideals, maximum weights.

\begin{abstract}
We introduce a new construction based on directed graphs. It provides a common generalization of the incidence rings and Munn semirings. Our main theorem describes all ideals of the largest possible weight in this construction. Several previous results can be obtained as corollaries to our new main theorem.
\end{abstract}

\section{Introduction}

Many interesting results have been obtained recently in the literature devoted to properties of graphs (cf. $[12,13,14,15,16,21,28,33])$ as well as algebraic constructions (cf. [22, 25, 27, 29, 31]). Incidence algebras of directed graphs have been studied for a long time (cf. [24, 31]). On the other hand, Munn semirings have also been considered by several authors too (cf. [9]). They were defined by analogy with the Munn algebras, which are very well known (cf. [30]).

The present paper introduces Munn incidence semirings as a common generalization of the incidence algebras and Munn semirings. A complete definition of this new construction is given in the section "Main Results" below. The main theorem of this paper is Theorem 1 in the same section. It describes all ideals of the largest possible weight in Munn incidence semirings. This result belongs to the large area studying the weights of ideals in various constructions. For instance, the ideals of the largest possible weight have been described for certain classes of the polynomial quotient rings in [26], structural matrix semirings in [17], Munn semirings in [9], and incidence semirings in [8, 10]. The investigation of ideals with largest weight has been motivated by applications of ideals in data mining (cf. $[8,11,25]$ ), health informatics (cf. $[4,6,7,19,20,32])$ and information security (cf. [1, 2, 5]).

It is important to describe the weights of ideals in the new and more general construction of the Munn incidence semirings for the following reasons. First, the ideals in a more general construction may lead to a discovery of systems with better properties crucial for cryptographic applications. Second, it is nice to unify already known facts in such a way that several previous results can be derived as corollaries to a new and more general theorem. In particular, proofs of the main results of the previous papers [8], and [10] can now be derived from the main theorem of the present article.

\section{Preliminaries}

We use standard terminology and refer the readers to $[4,13,18,22,23,31]$ for more detailed explanations of notions used in the present article. Throughout the word 'digraph' means a directed graph without multiple parallel edges but possibly with loops, and $D=(V, E)$ is a digraph with the set $V$ of vertices and the set $E$ of edges. The set of all positive integers is denoted by $\mathbb{N}$. 
Here we use the standard definition of a semiring without assuming that every semiring contains an identity element, see [9] and [17].

For any subset $S$ of a semiring $Q$, the ideal generated by $S$ in $Q$ is denoted by $\operatorname{id}(S)$ and is defined as the set of all sums of these elements and their multiples, i.e.,

$$
\mathbf{i d}(S)=\left\{\sum_{i=1}^{m} \sum_{j=1}^{m_{i}} \ell_{i j} s_{i} r_{i j} \mid s_{i} \in S, \ell_{i j}, r_{i j} \in Q \cup\{1\}\right\} .
$$

It is easily seen that the set $\operatorname{id}(S)$ is a subsemiring of $Q$ closed for the multiplication by the elements of $Q$, i.e., id $(S) Q+Q \mathbf{i d}(S) \subseteq \mathbf{i d}(S)$.

Recall that a semiring $Q$ is said to be idempotent if $x+x=x$ for all $x \in Q$. A semiring $Q$ is said to be zero-divisor-free, if $x y=0$ implies $x=0$ or $y=0$, for any $x, y \in Q$.

Let $Q$ be a semiring, $I$ and $\Lambda$ finite nonempty sets, and let $P$ be a $\Lambda \times I$-matrix with entries in $Q$. A Munn semiring over $Q$ with sandwich-matrix $P$ is the set $M(Q ; I, \Lambda ; P)$, consisting of all $I \times \Lambda$ matrices over $Q$, equipped with the usual addition and multiplication $\cdot$ defined by $A \cdot B=A P B$, for $A, B \in M(Q ; I, \Lambda ; P)$.

The concept of an incidence semiring was defined in [8] as an exact analogue of an incidence algebra. Let $Q$ be a semiring. The incidence algebra of $D=(V, E)$ over $Q$ is denoted by $I_{D}(Q)$ and is defined as the set consisting of zero 0 and all finite sums $\sum_{i=1}^{n} q_{i}\left(u_{i}, v_{i}\right)$, where $n \geq 1,0 \neq q_{i} \in Q$, $\left(u_{i}, v_{i}\right) \in E$, endowed with the usual addition and with multiplication defined by the distributive law and the rule

$$
\left(u_{1}, v_{1}\right) \cdot\left(u_{2}, v_{2}\right)= \begin{cases}\left(u_{1}, v_{2}\right) & \text { if } v_{1}=u_{2} \text { and }\left(u_{1}, v_{2}\right) \in E, \\ 0 & \text { otherwise }\end{cases}
$$

for all $\left(u_{1}, v_{1}\right),\left(u_{2}, v_{2}\right) \in E$, see $[22,31]$.

\section{Main Results}

Let $D=(V, E)$ be a digraph with the (possibly infinite) set $V$ of vertices and set $E$ of edges, and let $I$ and $\Lambda$ be nonempty sets such that $V=I \cup \Lambda$. Let $Q$ be a semiring, and let $P=\left[p_{\lambda i}\right]$ be a $(\Lambda \times I)$-matrix with entries $p_{\lambda i} \in Q$, for all $\lambda \in \Lambda, i \in I$. For $i \in I, \lambda \in \Lambda$, denote by $e_{i \lambda}$ the standard elementary $I \times \Lambda$ matrix with 1 in the intersection of $i$-th row and $\lambda$-th column and zeros in all other entries. Denote by $M_{D}(Q ; I, \Lambda ; P)$ the set of all $I \times \Lambda$ matrices $x$ of the form

$$
x=\sum_{j=1}^{n} x_{i_{j} \lambda_{j}} e_{i_{j} \lambda_{j}}
$$

where $\left(i_{j}, \lambda_{j}\right) \in E \cap(I \times \Lambda)$ and $0 \neq x_{i_{j} \lambda_{j}} \in Q$, for $j=1, \ldots, n$. Endow this set with the usual componentwise addition of matrices and with a multiplication - defined by the distributive laws and the rule

$$
x_{i_{1} \lambda_{1}} e_{i_{1} \lambda_{1}} \cdot x_{i_{2} \lambda_{2}} e_{i_{2} \lambda_{2}}= \begin{cases}\left(x_{i_{1} \lambda_{1}} p_{\lambda_{1} i_{2}} x_{i_{2} \lambda_{2}}\right) e_{i_{1} \lambda_{2}} & \text { if }\left(i_{1}, \lambda_{2}\right) \in E \\ 0 & \text { otherwise }\end{cases}
$$

for any $\left(i_{1}, \lambda_{1}\right),\left(i_{2}, \lambda_{2}\right) \in E \cap(I \times \Lambda)$ and any $x_{i_{1} \lambda_{1}}, x_{i_{2} \lambda_{2}} \in Q$. If the multiplication defined by (4) is associative, then we say that $M_{D}(Q ; I, \Lambda ; P)$ is a Munn incidence semiring of the digraph $D$. Lemmas 2 and 3 in Section show that Munn incidence semirings of digraphs are a common generalisation of the standard Munn semirings and incidence algebras, including the incidence algebras of infinite digraphs.

Let $M_{D}(Q ; I, \Lambda ; P)$ be a Munn incidence semiring of the digraph $D=(V, E)$. For each element $x$ in $M_{D}(Q ; I, \Lambda ; P)$, the weight $\mathbf{w t}(x)$ of $x$ is equal to the number of nonzero coefficients $x_{i_{j} \lambda_{j}}$ in 
the finite sum (3) for $x$ (cf. [23, $§ 2.13]$ ). If $S$ is a subset of $M_{D}(Q ; I, \Lambda ; P)$, then the weight of $S$ is denoted by $\mathbf{w t}(S)$ and is defined as the minimum weight of a nonzero element in $S$.

For any subset $S$ of $F=M_{D}(Q ; I, \Lambda ; P)$ and any $i \in I, \lambda \in \Lambda, A \subseteq E, X \subseteq I, Y \subseteq \Lambda$, we use the following notation: $S_{i \lambda}=\left\{q e_{i \lambda} \in S \mid q \in Q\right\}, S_{A}=S \cap \sum_{(i, \lambda) \in A} F_{i \lambda}, S_{X Y}=S \cap \sum_{i \in X, \lambda \in Y} F_{i \lambda}$, $S_{X *}=S \cap \sum_{i \in X, \lambda \in \Lambda} F_{i \lambda}, S_{* Y}=S \cap \sum_{i \in I, \lambda \in Y} F_{i \lambda}$. Besides, we put $S_{X \lambda}=S_{X\{\lambda\}}, S_{i Y}=S_{\{i\} Y}$, $S_{i *}=S_{\{i\} *}, S_{* \lambda}=S_{*\{\lambda\}}$ and assume that $S_{\emptyset Y}=S_{X \emptyset}=S_{\emptyset *}=S_{* \emptyset}=S_{\emptyset}=S_{\emptyset \emptyset}=0$.

We introduce the following sets of edges

$$
\begin{aligned}
& E_{L}=\left\{(u, v) \in E \mid \forall w, x \in V:(w, x),(w, v) \in E \Rightarrow p_{x u}=0\right\} \\
& E_{R}=\left\{(u, v) \in E \mid \forall w, x \in V:(w, x),(u, x) \in E \Rightarrow p_{v w}=0\right\} .
\end{aligned}
$$

For any vertex $v \in V$, define the following sets of vertices:

$$
\begin{aligned}
\operatorname{In}(v) & =\{i \in I \mid(i, v) \in E\}, \\
\operatorname{In}_{P}(v) & =\left\{i \in I \mid \exists t: p_{t v} \neq 0,(i, t) \in E\right\}, \\
\operatorname{Out}(v) & =\{\lambda \in \Lambda \mid(v, \lambda) \in E\}, \\
\operatorname{Out}_{P}(v) & =\left\{\lambda \in V \mid \exists x: p_{v x} \neq 0,(x, \lambda) \in E\right\} .
\end{aligned}
$$

For any $m \in \mathbb{N}$, denote by $\mathcal{K}_{L}(m)$ the set of all pairs $(Y, \lambda)$, where $\lambda \in \Lambda$ and $Y \subseteq \operatorname{In}(\lambda)$ are such that $|Y|=m,(i, \lambda) \in E_{L}$ for all $i \in Y$, and the intersection $\operatorname{Out}(i) \cap \operatorname{Out}_{P}(\lambda)$ is equal to the same set for all $i \in Y$, i.e., $\operatorname{Out}\left(i_{1}\right) \cap \operatorname{Out}_{P}(\lambda)=\operatorname{Out}\left(i_{2}\right) \cap \operatorname{Out}_{P}(\lambda)$, for all $i_{1}, i_{2} \in Y$.

Likewise, denote by $\mathcal{K}_{F}(m)$ the set of all pairs $(i, Y)$, where $i \in I$ and $Y \subseteq$ Out $(i)$ are such that $|Y|=m,(i, \lambda) \in E_{R}$ for all $\lambda \in Y$, and the intersection $\operatorname{In}_{P}(i) \cap \operatorname{In}(\lambda)$ is equal to the same set for all $\lambda \in Y$, i.e., $\operatorname{In}_{P}(i) \cap \operatorname{In}\left(\lambda_{1}\right)=\operatorname{In}_{P}(i) \cap \operatorname{In}\left(\lambda_{2}\right)$, for all $\lambda_{1}, \lambda_{2} \in Y$.

Further, we assume that $D=(V, G)$ is a finite digraph. Then the cardinality $N_{Z}=\left|E_{R} \cap E_{L}\right|$ is an integer. Besides, denote by $N_{L}$ the largest positive integer such that the set $\mathcal{K}_{L}\left(N_{L}\right)$ is not empty, and put $N_{L}=0$ if such integers do not exist. Likewise, denote by $N_{R}$ the largest positive integer such that the set $\mathcal{K}_{F}\left(N_{R}\right)$ is not empty, and put $N_{R}=0$ if such integers do not exist.

Let us define the following subsets of the Munn incidence semiring $M_{D}(Q ; I, \Lambda ; P)$ :

$$
\begin{aligned}
& \mathcal{A}_{L}=\left\{\sum_{i \in Y} q_{i \lambda} e_{i \lambda} \mid(Y, \lambda) \in \mathcal{K}_{L}\left(N_{L}\right), 0 \neq q_{i \lambda} \in Q \text { for all } i\right\}, \\
& \mathcal{A}_{R}=\left\{\sum_{\lambda \in Y} q_{i \lambda} e_{i \lambda} \mid(i, Y) \in \mathcal{K}_{R}\left(N_{R}\right), 0 \neq q_{i \lambda} \in Q \text { for all } \lambda\right\}, \\
& \mathcal{A}_{Z}=\left\{\sum_{(i, \lambda) \in E_{R} \cap E_{L}} q_{i \lambda} e_{i \lambda} \mid 0 \neq q_{i \lambda} \in Q \text { for all }(i, \lambda) \in E_{R} \cap E_{L}\right\} .
\end{aligned}
$$

Our main theorem describes ideals that have the largest possible weight among the weights of all ideals in Munn incidence semirings.

Theorem 1. Let $Q$ be a zero-divisor-free idempotent semiring with identity element, let $F$ be a Munn incidence semiring $M_{D}(Q ; I, \Lambda ; P)$ of the finite digraph $D=(V, E)$, and let $T$ be an ideal of $F$ such that $T$ has the largest possible weight. Then the following conditions are satisfied:

(a) if $\mathbf{w t}(T)>1$, then there exists a generator $x$ in $\mathcal{A}_{L} \cup \mathcal{A}_{R} \cup \mathcal{A}_{Z}$ such that $\mathbf{w t}(x)=\mathbf{w t}(T)$ and $x \in T$;

(b) $\mathbf{w t}(T)=\max \left\{1, N_{L}, N_{R}, N_{Z}\right\}$. 


\section{Technical Lemmas and Proofs}

Let us begin with a few technical properties of the Munn incidence semirings. The following two lemmas show that Munn incidence semirings of digraphs are a common generalization of the incidence algebras and Munn semirings.

Lemma 2. Every incidence algebra $I_{D}(Q)$ of a digraph $D=(V, E)$ is isomorphic to the Munn incidence semiring $M_{D}(Q ; V, V ; P)$ of the same digraph $D$, where $P$ is the identity $|V| \times|V|$-matrix.

Proof follows immediately from the definitions of the Munn incidence semiring and incidence algebra.

Lemma 3. Every $M(Q ; I, \Lambda ; P)$ is isomorphic to the Munn incidence semiring $M_{D}(Q ; I, \Lambda ; P)$ of the digraph $D$, where $D$ is the complete digraph on the set $I \cup \Lambda$ of vertices.

Proof follows immediately from the definitions of the Munn incidence semiring and classical Munn semiring.

We say that the digraph $D$ is $P$-balanced if, for all $i_{1}, \lambda_{1}, i_{2}, \lambda_{2}, i_{3}, \lambda_{3} \in V$ such that $\left(i_{1}, \lambda_{1}\right)$, $\left(i_{2}, \lambda_{2}\right),\left(i_{3}, \lambda_{3}\right),\left(i_{1}, \lambda_{3}\right) \in E \cap(I \times \Lambda)$ and $p_{\lambda_{1} i_{2}}, p_{\lambda_{2} i_{3}} \neq 0$, the following equivalence holds:

$$
\left(i_{1}, \lambda_{2}\right) \in E \Leftrightarrow\left(i_{2}, \lambda_{3}\right) \in E .
$$

Lemma 4. The set $M_{D}(Q ; I, \Lambda ; P)$ is a Munn incidence semiring of the digraph $D=(V, E)$ if and only if $D$ is P-balanced.

Proof. Suppose that the digraph $D$ is $P$-balanced. Then we are going to verify that the associative law $x(y z)=(x y) z$ is satisfied, for all $x, y, z \in M_{D}(Q ; I, \Lambda ; P)$. The distributive law and equality (3) imply that it is enough to consider the case where $x=q_{x} e_{i_{x} \lambda_{x}}, y=q_{y} e_{i_{y} \lambda_{y}}, z=q_{z} e_{i_{z} \lambda_{z}}$, for some $q_{x}, q_{y}, q_{z} \in Q$ and $\left(i_{x}, \lambda_{x}\right),\left(i_{y}, \lambda_{y}\right),\left(i_{z}, \lambda_{z}\right) \in E \cap(I \times \Lambda)$.

If $p_{\lambda_{x} i_{y}}=0$ or $p_{\lambda_{y} i_{z}}=0$, then we get $x(y z)=0=(x y) z$, as required. Likewise, if $\left(i_{x}, \lambda_{z}\right) \notin E$, then it follows that $x(y z)=0=(x y) z$, too. Further, we assume that $p_{\lambda_{x} i_{y}}, p_{\lambda_{y} i_{z}} \neq 0$ and $\left(i_{x}, \lambda_{z}\right) \in E$.

If $\left(i_{x}, \lambda_{y}\right) \notin E$, then (4) tells us that $x y=0$, and so $(x y) z=0$. Since $D$ is $P$-balanced, we get $\left(i_{y}, \lambda_{z}\right) \notin E$. Therefore $y z=0$ and $x(y z)=0$, and so the associative law holds.

It remains to consider the case where $\left(i_{x}, \lambda_{y}\right) \in E$. Then $\left(i_{y}, \lambda_{z}\right) \in E$, because $D$ is $P$-balanced. Therefore we get $(x y) z=\left(q_{x} p_{\lambda_{x} i_{y}} q_{y}\right) e_{i_{x} \lambda_{y}} \cdot z=\left(q_{x} p_{\lambda_{x} i_{y}} q_{y} p_{\lambda_{y} i_{z}} q_{z}\right) e_{i_{x} \lambda_{z}}=x \cdot\left(q_{y} p_{\lambda_{y} i_{z}} q_{z}\right) e_{i_{y} \lambda_{z}}=$ $x(y z)$. Thus, the associative law holds true, as required.

Conversely, let us assume that $M_{D}(Q ; I, \Lambda ; P)$ is a semiring. Then we have to verify that the equivalence (14) holds. By way of contradiction, suppose that there exist $i_{x}, \lambda_{x}, i_{y}, \lambda_{y}, i_{z}, \lambda_{z}$ such that $\left(i_{x}, \lambda_{x}\right),\left(i_{y}, \lambda_{y}\right),\left(i_{z}, \lambda_{z}\right),\left(i_{x}, \lambda_{z}\right) \in E \cap(I \times \Lambda)$ and $p_{\lambda_{x} i_{y}}, p_{\lambda_{y} i_{z}} \neq 0$.

If $\left(i_{x}, \lambda_{y}\right) \notin E$ and $\left(i_{y}, \lambda_{z}\right) \in E$, then (4) yields that $x y=0$, and so $(x y) z=0$. By (4), we get $x(y z) \neq 0$, a contradiction with the associative law.

On the other hand, if $\left(i_{x}, \lambda_{y}\right) \in E$ and $\left(i_{y}, \lambda_{z}\right) \notin E$, then (4) implies that $y z=0$; whence $x(y z)=0$. By (4), we get $(x y) z \neq 0$, a contradiction again. This completes the proof.

The following lemma is easy and well known.

Lemma 5. ([18]) Every idempotent semiring $Q$ is zero-sum-free, i.e., for all $q_{1}, \ldots, q_{n} \in Q$, the equality $q_{1}+\cdots+q_{n}=0$ implies that $q_{1}=\cdots=q_{n}=0$.

For any semiring $Q$, the left annihilator of $Q$ is the set $\operatorname{Ann}_{L}(Q)=\{x \in Q \mid x Q=0\}$, and the right annihilator of $Q$ is the set $\mathbf{A n n}_{R}(Q)=\{x \in Q \mid Q x=0\}$. It follows immediately that $\operatorname{Ann}_{L}(Q)$ and $\mathbf{A n n}_{R}(Q)$ are ideals of the semiring $Q$. 
Lemma 6. Let $Q$ be a zero-divisor-free idempotent semiring with identity element, and let $F$ be a Munn incidence semiring $M_{D}(Q ; I, \Lambda ; P)$ of the digraph $D=(V, E)$. Then $\operatorname{Ann}_{R}(F)=F_{E_{L}}$ and $\operatorname{Ann}_{L}(F)=F_{E_{R}}$.

Proof. Put $T_{R}=\mathbf{A n n}_{R}(F)$. First, let us prove the inclusion $T_{R} \supseteq F_{E_{L}}$. Suppose to the contrary that there exists a nonzero element $x$ in $F_{E_{L}}$ such that $x \notin T_{R}$. Then we have $F x \neq 0$, and so there exists $q e_{i \lambda} \in F$ such that $q e_{i \lambda} x \neq 0$. The definition of $F_{E_{L}}$ and equality (3) show that $x=\sum_{j=1}^{n} q_{j} e_{i_{j} \lambda_{j}}$, for some $n>0,0 \neq q_{j} \in Q, q_{j} \in Q,\left(i_{j}, \lambda_{j}\right) \in E_{L}$. It follows that $q e_{i \lambda} q_{j} e_{i_{j} \lambda_{j}} \neq 0$, for some $j$. Hence (4) yields that $p_{\lambda i_{j}} \neq 0$ and $\left(i, \lambda_{j}\right) \in E$. Therefore (5) implies that $\left(i_{j}, \lambda_{j}\right) \notin E_{L}$. This contradicts the choice of $x$ in $F_{E_{L}}$ and shows that $x$ belongs to $T_{R}$. Thus, $T_{R} \supseteq F_{E_{L}}$.

Second, let us prove the reversed inclusion. Choose any element $x$ in $T_{R}$. Equality (3) implies that $x=\sum_{j=1}^{n} q_{j} e_{i_{j} \lambda_{j}}$, for some $n>0,0 \neq q_{j} \in Q, q_{j} \in Q,\left(i_{j}, \lambda_{j}\right) \in E$. Suppose to the contrary that $x \notin F_{E_{L}}$.

Then the definition of $F_{E_{L}}$ shows that there exists $j$ such that $\left(i_{j}, \lambda_{j}\right)$ belongs to $E \backslash E_{L}$. It follows from (5) that there exists $(w, \mu)$ in $E$ such that $\left(w, \lambda_{j}\right) \in E$ and $p_{\mu i_{j}} \neq 0$. Therefore (4) yields that $e_{w \mu}\left(q_{j} e_{i_{j} \lambda_{j}}\right)=\left(p_{\mu i_{j}} q_{j}\right) e_{w, \lambda_{j}} \neq 0$ in $F$, where $p_{\mu i_{j}} q_{j} \neq 0$ because $Q$ is zero-divisor-free. However, $e_{w \mu} q_{j} e_{i_{j} \lambda_{j}}$ is a summand of $e_{w \mu} x$ with coefficient $q_{j}$. Since $Q$ is an idempotent semiring, Lemma 5 shows that this summand does not cancel with other summands of $e_{w \mu} x$. Therefore $e_{w \mu} x \neq 0$. This contradicts the choice of $x$ in $T_{R}$, and shows that $x$ belongs to $F_{E_{L}}$.

Thus, $T_{R}=F_{E_{L}}$, as required. The proof of the second equality $\operatorname{Ann}_{L}(F)=F_{E_{R}}$ is dual and we omit it.

Lemma 7. Let $Q$ be a zero-divisor-free idempotent semiring with identity element, and let $F$ be a Munn incidence semiring $M_{D}(Q ; I, \Lambda ; P)$ of the digraph $D=(V, E)$. Then $\mathbf{w t}(\mathbf{i d}(x))=\mathbf{w t}(x)=$ $N_{L}$, for all $x \in \mathcal{A}_{L}$.

Proof. Choose any element $x$ in $\mathcal{A}_{L}$. In view of (11), we can write it down as $x=\sum_{i \in Y} q_{i \lambda} e_{i \lambda}$, for some $(Y, \lambda) \in \mathcal{K}_{L}\left(N_{L}\right)$ and $0 \neq q_{i \lambda} \in Q$. The definition of $\mathcal{K}_{L}\left(N_{L}\right)$ yields that $|Y|=N_{L}$. Thus, we have $\mathbf{w t}(x)=|Y|=N_{L}$.

To prove that $\mathbf{w t}(\mathbf{i d}(x))=\mathbf{w t}(x)$, choose a nonzero element $y$ in $\operatorname{id}(x)$. The inequality $\mathbf{w t}(\mathbf{i d}(x)) \leq \mathbf{w t}(x)$ is obvious, and so it remains to verify that $\mathbf{w t}(y) \geq \mathbf{w t}(x)$.

It follows from (1) that $y$ can be written down in the form $y=\sum_{j=1}^{k} \ell_{j} x r_{j}$, for some $\ell_{j}, r_{j} \in$ $F \cup\{1\}$. We may assume that only nonzero summands $\ell_{j} x r_{j}$ have been included in representation for $y$ given above. The definition of $\mathcal{K}_{L}\left(N_{L}\right)$ tells us that $(i, \lambda) \in E_{L}$, for all $i \in Y$. Lemma 6 shows that $x$ belongs to $\mathbf{A n n}_{R}(F)$. This forces all products $\ell_{j} x$ to be equal to zero whenever $\ell_{j}$ is in $F$. It follows that $\ell_{1}=\cdots=\ell_{k}=1$.

In view of (3) each element $r_{j} \in F$, that occurs in the expression for $y$ given above, can be represented in the form $r_{j}=\sum_{a=1}^{b} h_{j}(a) e_{i_{j}(a) \lambda_{j}(a)}$, for some $b \in \mathbb{N}, h_{j}(a) \in Q, i_{j}(a) \in I, \lambda_{j}(a) \in \Lambda$. Substituting these representations in the sum for $y$ given above and applying the distributive law, to simplify notation we may assume that from the very beginning each element $r_{j} \neq 1$ itself has the form $r_{j}=h_{j} e_{i_{j} \lambda_{j}}$, for some $0 \neq h_{j} \in Q,\left(i_{j}, \lambda_{j}\right) \in E$.

Given that $x \in \mathcal{A}_{L}$ and $(Y, \lambda) \in \mathcal{K}_{L}\left(N_{L}\right)$, the last condition in the definition of $\mathcal{K}_{L}\left(N_{L}\right)$ tells us that the intersection $\operatorname{Out}(i) \cap \operatorname{Out}_{P}(\lambda)$ is equal to one and the same set for all $i \in Y$, i.e., $\operatorname{Out}\left(i_{1}\right) \cap$ $\operatorname{Out}_{P}(\lambda)=\operatorname{Out}\left(i_{2}\right) \cap \operatorname{Out}_{P}(\lambda)$, for all $i_{1}, i_{2} \in Y$. Since $x r_{j} \neq 0$, it follows from (4) that $p_{\lambda i_{j}} \neq 0$ and that there exists $i_{1}$ in $Y$ such that $\lambda_{j}$ belongs to $\operatorname{Out}\left(i_{1}\right) \cap \operatorname{Out}_{P}(\lambda)$. Consequently, $\lambda_{j}$ is in Out $(i) \cap$ Out $_{P}(\lambda)$, for all $i \in Y$.

Fix any $i \in Y$ and consider the summand $q_{i} e_{i \lambda}$ of $x$. Since $Q$ is zero-divisor-free and $p_{\lambda i_{j}} \neq 0$, we get $q_{i} p_{\lambda i_{j}} h_{j} \neq 0$. Hence (4), (9) and (10) imply that $q_{i} e_{i \lambda} \cdot r_{j}=q_{i} e_{i \lambda} \cdot h_{j} e_{i_{j} \lambda_{j}}=\left(q_{i} p_{\lambda i_{j}} h_{j}\right) e_{i \lambda_{j}} \neq 0$.

It follows that $\mathbf{w t}\left(x r_{j}\right)=|Y|=\mathbf{w t}(x)$, for each $r_{j} \neq 1$. The equality $\mathbf{w t}\left(x r_{j}\right)=\mathbf{w t}(x)$ also holds trivially for each $r_{j}=1$ in the sum for $y$. Since $Q$ is an idempotent semiring, it follows from Lemma 5 that $\mathbf{w t}(y) \geq \mathbf{w t}(x)$. By the choice of $x$, this means that $\mathbf{w t}(\mathbf{i d}(x))=\mathbf{w t}(x)$. This completes the proof. 
Lemma 8. Let $Q$ be a zero-divisor-free idempotent semiring with identity element, and let $F$ be a Munn incidence semiring $M_{D}(Q ; I, \Lambda ; P)$ of the digraph $D=(V, E)$. Then $\mathbf{w t}(\mathbf{i d}(x))=\mathbf{w t}(x)=$ $N_{R}$, for all $x \in \mathcal{A}_{R}$.

Proof. The proof of Lemma 8 is dual to the proof of Lemma 7 and we omit it.

Lemma 9. Let $Q$ be a zero-divisor-free idempotent semiring with identity element, and let $F$ be a Munn incidence semiring $M_{D}(Q ; I, \Lambda ; P)$ of the digraph $D=(V, E)$. Then $\mathbf{w t}(x)=\mathbf{w t}(\mathbf{i d}(x))=$ $N_{Z}$, for all $x \in \mathcal{A}_{Z}$.

Proof. Take any element $x$ in $\mathcal{A}_{Z}$. By (13), it can be recorded in the form $x=\sum_{(i, \lambda) \in E_{R} \cap E_{L}} q_{i \lambda} e_{i \lambda}$, for some $0 \neq q_{i \lambda} \in Q$. Clearly, wt $(x)=\left|E_{R} \cap E_{L}\right|=N_{Z}$. Choose a nonzero element $y$ in id $(x)$. It follows from (1) that $y$ can be written down as $y=\sum_{j=1}^{k} \ell_{j} x r_{j}$, for some $\ell_{j}, r_{j} \in F \cup\{1\}$.

Since $Q$ is an idempotent semiring with identity element, Lemma 6 tells us that $\operatorname{Ann}_{R}(F)=F_{E_{L}}$ and $\mathbf{A n n}_{L}(F)=F_{E_{R}}$. Hence we get $x \in F_{E_{L} \cap E_{R}}=F_{E_{L}} \cap F_{E_{R}}=\mathbf{A n n}_{R}(F) \cap \mathbf{A n n}_{L}(F)$. It follows that if $\ell_{j} \in F$ or $r_{j} \in F$, then $\ell_{j} x r_{j}=0$ in the representation for $y$. Therefore we may assume that $\ell_{j}=r_{j}=1$, for all summands of the representation of $y$ given above. This means that $\mathbf{i d}(x)=\mathbb{N} x$.

Now, take any element $k x \neq 0$ in $\mathbb{N} x$, where $k \in \mathbb{N}$. Suppose that $k q_{i \lambda}=0$ for some summand $q_{i \lambda} e_{i \lambda}$ of $x$. Since $Q$ has an identity element $1_{Q}$, we infer that $k 1_{Q}=k x \neq 0$ in $Q$. The equalities $\left(k 1_{Q}\right) q_{i \lambda}=k q_{i \lambda}=0$ mean that $k 1_{Q}$ is a zero divisor in $Q$. This contradicts the hypothesis that $Q$ is zero-divisor-free. It follows that, for every $k \in \mathbb{N}$ such that $k x \neq 0$, the weight of $k x$ is equal to $\mathbf{w t}(x)$. We conclude that $\mathbf{w t}(\mathbf{i d}(x))=\mathbf{w t}(x)$, which completes the proof.

Proof of Theorem 1. To prove condition (a) we suppose that $\mathbf{w t}(T)>1$. Choose a nonzero element $x$ of minimal weight in $T$. Then we have $\mathbf{w t}(x)=\mathbf{w t}(T)>1$. Lemma 6 says that $\mathbf{A n n}_{L}(F)=$ $F_{E_{R}}$ and $\mathbf{A n n}_{R}(F)=F_{E_{L}}$.

If $x \notin \mathbf{A n n}_{R}(F) \cup \mathbf{A} \mathbf{n n}_{L}(F)$, then there exist $q_{1} e_{i_{1} \lambda_{1}}$ and $q_{2} e_{i_{2} \lambda_{2}}$ in $F$ such that the products $q_{1} e_{i_{1} \lambda_{1}} x$ and $x q_{2} e_{i_{2} \lambda_{2}}$ are nonzero. Equality (4) yields us that the product $y=q_{1} e_{i_{1} \lambda_{1}} x q_{2} e_{i_{2} \lambda_{2}}$ is nonzero too. Since $Q$ is zero-divisor-free, it also follows from (4) that $y \in F_{i_{1} \lambda_{2}}$; whence $\mathbf{w t}(y)=1$. However, $y$ belongs to $T$. This contradicts the hypothesis that $\mathbf{w t}(T)=1$, and shows that $x$ always belongs to the union $\mathbf{A n n}_{R}(F) \cup \mathbf{A} \mathbf{n n}_{L}(F)$. Therefore the following three cases may occur.

Case 1. $x \in \operatorname{Ann}_{R}(F) \cap \mathbf{A n n}_{L}(F)$. Then $x \in F_{E_{L}} \cap F_{E_{R}}=F_{E_{L} \cap E_{R}}$, and so $\mathbf{w t}(T)=\mathbf{w t}(x) \leq$ $N_{Z}$. On the other hand, it follows from the maximality of $\mathbf{w t}(T)$ and Lemma 9 that $\mathbf{w t}(T) \geq N_{Z}$. Therefore $\mathbf{w t}(x)=N_{Z}$. Hence (13) shows that $x \in \mathcal{A}_{Z}$, which means that condition (a) holds.

Case 2. $x \in \operatorname{Ann}_{R}(F) \backslash \operatorname{Ann}_{L}(F)$. Then $x \in F_{E_{L}}$. In view of (3), we infer

$$
x=\sum_{j=1}^{n} q_{j} e_{i_{j} \lambda_{j}}
$$

where $n \geq 0,0 \neq q_{j} \in Q$, and $\left(i_{j}, \lambda_{j}\right) \in E_{L}$. Since $x$ does not belong to $\operatorname{Ann}_{L}(F)$, it follows that there exists $q e_{i \lambda} \in F$ such that $x q e_{i \lambda} \neq 0$.

Consider the set $Y=\left\{i_{1}, \ldots, i_{n}\right\}$. We are going to verify that the pair $(Y, \lambda)$ satisfies all conditions in the definition of $\mathcal{K}_{L}(n)$.

First, note that $\lambda \in \Lambda$, as required in the definition of $\mathcal{K}_{L}(n)$.

Second, observe that the inequality $\mathbf{w t}\left(x q e_{i \lambda}\right) \leq \mathbf{w t}(x)$ is always true in $F$. The reversed inequality follows from the minimality of $\mathbf{w t}(x)$ in $T$, because $x q e_{i \lambda} \in T$. Therefore $\mathbf{w t}\left(x q e_{i \lambda}\right)=\mathbf{w t}(x)$. This and (15) yield us that $q_{j} e_{i_{j} \lambda_{j}} \cdot q e_{i \lambda} \neq 0$, for all $j=1, \ldots, n$. By (4), $p_{\lambda_{j} i} \neq 0$ and $\left(i_{j}, \lambda\right) \in E$, for all $j=1, \ldots, n$. Therefore $Y \subseteq \operatorname{In}(\lambda)$, as required in the definition of $\mathcal{K}_{L}(n)$.

Third, given that $x$ belongs to the ideal $\mathbf{A n n}_{R}(F)$ of $F$, it is clear that $x q e_{i \lambda}$ is in $\operatorname{Ann}_{R}(F)$, too. Hence $\left(i_{j}, \lambda\right) \in E_{L}$, for all $i_{j} \in Y$, by Lemma 6 .

Fourth, it is clear that $|Y|=n$, as required in the definition of $\mathcal{K}_{L}(n)$, too. 
Fifth, to prove the last property required in the definition of $\mathcal{K}_{L}(n)$, suppose to the contrary that the intersections Out $\left(i_{1}\right) \cap \operatorname{Out}_{P}(\lambda)$ and $\operatorname{Out}\left(i_{2}\right) \cap \operatorname{Out}_{P}(\lambda)$ are different for some $i_{1}, i_{2}$ in $Y$. Without loss of generality we may assume that there exists $\mu \in \Lambda$ that belongs to $\operatorname{Out}\left(i_{1}\right) \cap \operatorname{Out}_{P}(\lambda)$ but does not belong to $\operatorname{Out}\left(i_{2}\right)$. Then $\left(i_{1}, \mu\right) \in E$ and $\left(i_{2}, \mu\right) \notin E$. Since $\mu \in \operatorname{Out}_{P}(\lambda)$, by (10) there exists $j \in I$ such that $p_{\lambda j} \neq 0$ and $(j, \mu) \in E$. Since $Q$ is a semiring with identity element, we have $e_{j \mu} \in F$. Therefore (4) implies that $q_{1} e_{i_{1} \lambda} e_{j \mu} \neq 0$, but $q_{1} e_{i_{1} \lambda} e_{j \mu}=0$. It follows that $\mathbf{w t}\left(x e_{j \mu}\right)<\mathbf{w t}(x)$. This contradicts the minimality of $\mathbf{w t}(x)$ in $T$, because $x e_{j \mu} \in T$. The contradiction shows that all intersections $\operatorname{Out}\left(i_{j}\right) \cap \mathbf{O u t}_{P}(\lambda)$ are equal to one and the same set, for all $i_{j} \in Y$. Thus, we have proved that $(Y, \lambda)$ belongs to $\mathcal{K}_{L}(n)$.

Further, the maximality of $N_{L}$ implies that $|Y| \leq N_{L}$. It follows from (15) that $x q e_{i \lambda} \in F_{Y \lambda}$. Hence we get $\mathbf{w t}(x)=\mathbf{w t}\left(x q e_{i \lambda}\right) \leq|Y| \leq N_{L}$. On the other hand, the maximality of $\mathbf{w t}(T)$ and Lemma 7 show that $\mathbf{w t}(T) \geq N_{L}$. Hence $\mathbf{w t}(x) \geq N_{L}$. Therefore $\mathbf{w t}\left(x q e_{i \lambda}\right)=\mathbf{w t}(x)=N_{L}$, and so $|Y|=N_{L}$. Thus, we conclude that $n=\mathbf{w t}(x)=N_{L}$ and $(Y, v) \in \mathcal{K}_{L}\left(N_{L}\right)$. By (11), we get $x(g ; i, \lambda) \in \mathcal{A}_{L}$. Therefore condition (a) holds in this case too.

Case 3. $x \in \mathbf{A n n}_{L}(F) \backslash \mathbf{A n n}_{R}(F)$. Then the proof is dual to the proof of Case 2, and so condition (a) holds again. This concludes the proof of condition (a).

To verify condition (b), first note that the inequality $\mathbf{w t}(T) \geq \max \left\{N_{L}, N_{R}, N_{Z}\right\}$ follows immediately from Lemmas 7,8 and 9 and the maximality of $\mathbf{w t}(T)$. If $\mathbf{w t}(T)=1$, then we get $N_{L}, N_{R}, N_{Z} \leq$ 1 ; whence condition (b) is met. On the other hand, if $\mathbf{w t}(T)>1$, then condition (a) implies that $\mathbf{w t}(T) \leq \max \left\{N_{L}, N_{R}, N_{Z}\right\}$, and so (b) holds again. This completes the proof.

\section{Acknnowledgements}

The first author was supported by Discovery grant DP0449469 from Australian Research Council. The second author was supported by ARC Discovery grant DP130101383. The third author was supported by Discovery grant DP0450294.

\section{References}

[1] J. Abawajy, G. Beliakov, A. Kelarev and M. Chowdhury, Iterative construction of hierarchical classifiers for phishing website detection, Journal of Networks 9 (2014), 2089-2098.

[2] J. Abawajy and A. Kelarev, A multi-tier ensemble construction of classifiers for phishing email detection and filtering, Cyberspace Safety and Security, CSS 2012, Lecture Notes in Computer Science 7672 (2012), 48-56.

[3] J. Abawajy, A.V. Kelarev and M. Chowdhury, Power graphs: a survey, Electronic J. Graph Theory and Applications 1 (2013), 125-147.

[4] J.H. Abawajy, A.V. Kelarev and M. Chowdhury, Multistage approach for clustering and classification of ECG data, Computer Methods and Programs in Biomedicine 112 (2013), 720-730.

[5] J. Abawajy, A. Kelarev and M. Chowdhury, Large iterative multitier ensemble classifiers for security of big data, IEEE Transactions on Emerging Topics in Computing 2 (2014), 352-363.

[6] J. Abawajy, A. Kelarev, M.U. Chowdhury and H.F. Jelinek, Enhancing predictive accuracy of cardiac autonomic neuropathy using blood biochemistry features and iterative multi-tier ensembles, IEEE J. Biomedical Health Informatics 20 (2016), 408-415. 
[7] J. Abawajy, A. Kelarev, M. Chowdhury, A. Stranieri and H.F. Jelinek, Predicting cardiac autonomic neuropathy category for diabetic data with missing values, Computers in Biology and Medicine 43 (2013), 1328-1333.

[8] J. Abawajy, A.V. Kelarev, M. Miller and J. Ryan, Incidence semirings of graphs and visible bases, Bull. Aust. Math. Soc. 89 (2014), 451-459.

[9] J. Abawajy, A.V. Kelarev and J. Zeleznikow, Optimization of classification and clustering systems based on Munn semirings, Semigroup Forum 87 (2013), 617-626.

[10] J. Abawajy, A.V. Kelarev, J.L. Yearwood and C. Turville, A data mining application of the incidence semirings, Houston J. Math. 39 (2013), 1083-1093.

[11] J. Abawajy, A.V. Kelarev, M. Miller, J. Ryan, Rees semigroups of digraphs for classification of data, Semigroup Forum 92 (2016), 121-134.

[12] M. Afkhami, M. Karimi and K. Khashyarmanesh, On the regular digraph of ideals of commutative rings, Bull. Aust. Math. Soc. 88 (2013), 177-189.

[13] M. Baca and M. Miller, Super Edge-Antimagic Graphs: A Wealth of Problems and Some Solutions, Brown Walker Press, 2008.

[14] B. Basavanagoud and V.R. Desai, Forgotten topological index and hyper-zagreb index of generalized transformation graphs, Bulletin of Mathematical Sciences and Applications 14 (2016), $1-6$.

[15] B. Basavanagoud and J.B. Veeragoudar, A criterion for (non-)planarity of the blocktransformation graph $G^{\alpha \beta \gamma}$ when $\alpha \beta \gamma=101$, Bulletin of Mathematical Sciences and Applications 10 (2014), 38-47.

[16] B. Curtin and G.R. Pourgholi, A group sum inequality and its application to power graphs, Bull. Aust. Math. Soc. 90 (2014), 418-426.

[17] D.Y. Gao, A.V. Kelarev and J.L. Yearwood, Optimization of matrix semirings for classification systems, Bull. Aust. Math. Soc. 84 (2011), 492-503.

[18] J.S. Golan, Semirings and Their Applications, Kluwer Academic Publishers, Dordrecht, 1999.

[19] H.F. Jelinek, J.H. Abawajy, A.V. Kelarev, M.U. Chowdhury and A. Stranieri, Decision trees and multi-level ensemble classifiers for neurological diagnostics, AIMS Medical Science 1 (2014), $1-12$.

[20] H.F. Jelinek, J.H. Abawajy, D. Cornforth, A. Kowalczyk, M. Negnevitsky, M.U. Chowdhury, R. Krones and A.V. Kelarev, Multi-layer attribute selection and classification algorithm for the diagnosis of cardiac autonomic neuropathy based on HRV attributes, AIMS Medical Science 2 (2015), 396-409.

[21] V.J. Kaneria, H.M. Makadia and R.V. Viradia, Graceful labeling for disconnected grid related graphs, Bulletin of Mathematical Sciences and Applications 11 (2015), 6-11.

[22] A.V. Kelarev, Ring Constructions and Applications, World Scientific, River Edge, NJ, 2002.

[23] A.V. Kelarev, Graph Algebras and Automata, Marcel Dekker, New York, 2003.

[24] A.V. Kelarev and D.S. Passman, A description of incidence rings of group automata, Contemporary Mathematics 456 (2008), 27-33. 
[25] A. Kelarev, J. Ryan and J. Yearwood, Cayley graphs as classifiers for data mining: the influence of asymmetries, Discrete Math. 309 (2009), 5360-5369.

[26] A.V. Kelarev, J.L. Yearwood and P.W. Vamplew, A polynomial ring construction for classification of data, Bull. Aust. Math. Soc. 79 (2009), 213-225.

[27] X.Q. Luo, On $J^{*}$-class of $\Pi^{*}$-regular semigroups, Bulletin of Mathematical Sciences and Applications 4 (2012), 1-5.

[28] A.N. Murugan, Magic graphoidal on class of trees, Bulletin of Mathematical Sciences and Applications 9 (2014), 33-44.

[29] C.J.S. Reddy, S.M. Rao and V.V. Kumar, Centralizing and commuting left generalized derivations on prime rings, Bulletin of Mathematical Sciences and Applications 11 (2015), 1-3.

[30] M. Soroushmehr, Homomorphisms of $\ell 1$-Munn algebras and applications to semigroup algebras, Bull. Aust. Math. Soc. 92 (2015), 115-122.

[31] E. Spiegel and C.J. O’Donnell, Incidence Algebras, Marcel Dekker, New York, 1997.

[32] A. Stranieri, J. Abawajy, A. Kelarev, S. Huda, M. Chowdhury and H.F. Jelinek, An approach for Ewing test selection to support the clinical assessment of cardiac autonomic neuropathy, Artificial Intelligence in Medicine, 58 (2013), 185-193.

[33] P. Sumathi and G. Alarmelumangai, Locating equitable domination and independence subdivision numbers of graphs, Bulletin of Mathematical Sciences and Applications 9 (2014), 27-32. 